\title{
Un nouvel habit pour une organisation forte
}

\author{
Anne-Geneviève Bütikofer
}

Secrétaire générale de la FMH

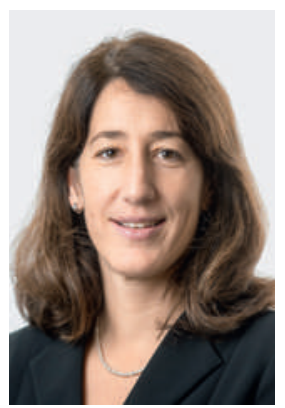

Avec plus de 39000 membres, la FMH est l'une des plus grandes associations professionnelles actives au plan national. Selon un sondage réalisé en 2014, aussi bien les médecins que les politiques, les représentants des autorités, les autres acteurs de la santé et les journalistes considèrent la FMH comme une organisation crédible, sérieuse et professionnelle, ces derniers reconnaissant également à la Fédération des médecins suisses une grande capacité de persuasion et une forte présence. Le succès d'une organisation ne repose cependant pas seulement sur la crédibilité de l'image qu'elle véhicule vis-à-vis de l'extérieur, mais également sur les objectifs qu'elle se fixe et qui reflètent les intérêts de ses membres. Ce n'est qu'en connaissant et en défendant les valeurs et les attentes de ces derniers qu'une organisation peut se profiler comme une association professionnelle fiable et comme un acteur de la santé digne de confiance.

Fort de ce constat, le Comité central de la FMH s'est fixé comme objectif stratégique pour la législature en cours de renforcer encore les services en faveur des membres. Pour ce faire, il s'est penché sur la manière d'optimiser les prestations, de répondre encore mieux aux besoins

\section{Une organisation professionnelle forte offre à ses membres une réelle plus-value et un profil unique.}

des membres et de leur offrir une réelle plus-value. En sa qualité de centre opérationnel de la FMH, le Secrétariat général a, dans cette perspective, répertorié et analysé de manière approfondie son activité. Il a ainsi pu présenter, pour la première fois, l'intégralité de ses prestations de manière synthétique dans un catalogue, et offre désormais un outil pratique et utile pour les membres.

La FMH a également profité du sondage évoqué plus haut pour se pencher sur son positionnement en tant qu'organisation professionnelle, et a demandé aux personnes interrogées de se prononcer sur la visibilité de la FMH, de l'Institut suisse pour la formation médicale postgraduée et continue (ISFM), organe autonome au sein de la FMH, et de l'Académie suisse pour la qualité en médecine (ASQM). A cette occasion, les trois groupes consultés - médecins, autres acteurs de la santé et grand public - ont clairement identifié les trois lettres «FMH» comme une marque faîtière connue et représentative d'une organisation crédible, sérieuse et professionnelle. La nouvelle identité visuelle de la FMH, qui se traduit par la nouvelle architecture entre le logo de la FMH, de sa sous-marque ASQM et de la marque de

\section{La FMH a harmonisé son image et se présente} avec une nouvelle identité visuelle à partir du mois de juin.

compétence ISFM, ancre encore mieux ce positionnement dans le paysage de la santé, et permet à la fois de respecter les particularités des différentes marques tout en reflétant leur appartenance commune. Au travers de ce nouvel ordonnancement et de ce lien visuel mais également de leurs nouvelles typographie et couleur, les nouveaux logos revêtent désormais une forme plus moderne, plus convaincante mais également plus innovante et assureront ainsi une pénétration du marché encore meilleure.

Le catalogue de prestations se présente déjà dans cette nouvelle parure, tout comme la lettre d'information électronique de la FMH, le «FMH-Flash», qui présentera désormais quatre fois par an les thèmes actuels en politique de la santé ainsi que les projets et travaux en cours à destination exclusive des membres. Grâce à des icônes spécifiques, les différentes rubriques du catalogue de prestations, du Flash et du rapport de gestion (disponible depuis 2014 dans sa nouvelle version) sont clairement identifiables, ce qui permet au lecteur de s'y retrouver plus facilement et plus rapidement. Ces pictogrammes seront également utilisés dans d'autres moyens de communication.

Les nouvelles identités visuelles de la FMH, de l'ISFM et de l'ASQM verront le jour en juin 2015. L'ISFM et l'ASQM pourront ainsi profiter de la grande visibilité de la marque faîtière FMH. En parallèle, le Secrétariat général poursuivra ce nouveau positionnement afin que les membres puissent encore mieux profiter de tous les avantages qui leur sont offerts. 